\title{
Localization and Blow-Up of Thermal Waves in Nonlinear Heat Conduction with Peaking
}

\author{
B. H. Gilding ${ }^{1}$ and M. A. Herrero ${ }^{2}$ \\ ${ }^{1}$ Enschede Faculty of Applied Mathematics, University of Twente, \\ 7500 AE Enschede, The Netherlands \\ ${ }^{2}$ Departamento de Matemática Aplicada, Facultad de Matemáticas, Universidad Complutense, \\ E-28040 Madrid, Spain
}

\section{Statement of Problem and Results}

Consider the initial-boundary value problem:

$$
\begin{aligned}
& u_{t}=\left(u^{m}\right)_{x x} \text { for }(x, t) \in(0, \infty) \times(0, T), \\
& u(x, 0)=u_{0}(x) \text { for } x \in[0, \infty) \text {, } \\
& u(0, t)=\psi(t) \quad \text { for } \quad t \in[0, T),
\end{aligned}
$$

where

$$
m>1, \quad 0<T<\infty,
$$

and $u_{0}$ and $\psi$ are given nonnegative continuous functions which satisfy the compatibility condition $u_{0}(0)=\psi(0)$. If $u_{0}$ is bounded, this problem is known to possess a unique solution $u(x, t)$ defined in some generalized sense. Moreover, if $u_{0}$ has compact support, for all $t \in[0, T)$ one can define the interface

$$
\zeta(t)=\sup \{x \in[0, \infty): u(x, t)>0\} .
$$

This interface constitutes a free boundary which is continuous and monotonic increasing on $[0, T)$.

In this paper, we study the behaviour of the solution $u(x, t)$ and the free boundary $\zeta(t)$ as $t \uparrow T$ under the hypothesis that

$$
\psi(t) \uparrow \infty \quad \text { as } \quad t \uparrow T
$$

Equation (1.1) is generally known as the porous media equation because of its description of the flow of a polytropic gas in a porous medium. However, the equation also arises in the study of a number of other physical problems [14]. One significant field of application of equation (1.1) is radiative heat transfer. In this context, $u$ denotes temperature and the equation describes the diffusion of heat in a one-dimensional medium where the thermal conductivity is an increasing power function of the temperature itself. The free boundary $\zeta(t)$ separates heated and cold regions of the medium and is termed a thermal front, while solutions which possess 
such a front are referred to as thermal waves [21]. Problem (1.1)-(1.3) under condition (1.4) is pertinent to combustion processes. It is said that when (1.4) holds, the boundary temperature of the conductive medium is in a peaking regime with peaking time, $T$. Of particular interest in this case are the phenomena of localization and of the formation of zones of heat intensification. Localization refers to the heated region of the medium remaining bounded (despite the fact that the boundary temperature becomes unbounded), whilst the formation of zones of heat intensification refers to the formation of entire regions in which the temperature tends to infinity at the peaking time. Cf. a series of articles appearing in Soviet Physics Doklady [10, 11, 15-18].

Henceforth, it will be supposed that the following hypotheses hold.

Hypotheses. The function $u_{0}$ is nonnegative, continuous, and has compact support on $[0, \infty)$. The function $\psi$ is nonnegative and continuous on $[0, T)$, and satisfies the compatibility condition $\psi(0)=u_{0}(0)$. Moreover, $\psi$ is monotonic increasing on $[0, T)$ and satisfies (1.4).

Localization will be said to occur if

$$
\limsup _{t \uparrow T} \zeta(t)<\infty
$$

Our first result is the following.

Theorem 1. Localization occurs if and only if

$$
\limsup _{t \uparrow T} \frac{\int_{0}^{t} \psi^{m}(s) d s}{\psi(t)}<\infty .
$$

Furthermore, if localization occurs then

$$
\limsup _{t \uparrow T}(T-t)^{1 /(m-1)} u(x, t)<\infty \text { for all } x>0,
$$

whereas, if localization does not occur then

$$
\limsup _{t T}(T-t)^{1 /(m-1)} u(x, t)=\infty \text { for all } x \geqq 0 .
$$

With regard to heat intensification, we adopt the mathematical convention of defining the blow-up set

$$
\Omega=\left\{x \in[0, \infty): \limsup _{t \uparrow T} u(x, t)=\infty\right\}
$$

We prove the following.

Theorem 2. If $x \in \Omega$ then

$$
\liminf _{t \uparrow T} u(x, t)=\infty .
$$

Moreover, $\Omega$ is a connected interval containing the point $x=0$.

It follows from Theorems 1 and 2 that if localization does not occur then $\Omega=[0, \infty)$. On the other hand, if localization does occur then $\Omega$ is a bounded interval containing the point $x=0$. 
Estimates of the size of the blow-up set constitute our remaining results. We let

$$
\omega=\sup \Omega,
$$

and introduce the constant

$$
\mu=2 m(m+1) /(m-1)^{2}
$$

and the real function $\chi$ defined on $[0,1]$ by

$$
\frac{1}{2} \mu \int_{0}^{\chi(\eta)}\left(1-\xi^{1 / 2}\right)^{2 /(m-1)} d \xi=\eta
$$

Note that $\chi$ is continuous, strictly monotonic increasing, and convex on $[0,1]$, with $\chi(0)=0$ and $\chi(1)=1$.

Theorem 3. Let $\phi(t)$ be a nontrivial, nonnegative, monotonic increasing, continuously-differentiable function on $(0, T)$, and let

$$
\begin{array}{ll}
A=\limsup _{t \uparrow T} \phi^{m}(t) / \phi^{\prime}(t), & a=\liminf _{t \uparrow T} \phi^{m}(t) / \phi^{\prime}(t), \\
B=\limsup _{t \uparrow T} \psi(t) / \phi(t), & b=\liminf _{t \uparrow T} \psi(t) / \phi(t),
\end{array}
$$

and

$$
C=\underset{t \uparrow T}{\limsup }\left[\int_{0}^{t} \psi^{m}(s) d s / \phi(t)\right] .
$$

(i) If $A<\infty$ and $B<\infty$ there holds $\omega^{2} \leqq \mu A B^{m-1}$.

(ii) If $a>0$ and $b>0$ there holds $\mu a b^{m-1} \leqq \omega^{2}$.

(iii) If $0<A<\infty$ and $0<B<\infty$ there holds $C \leqq A B^{m}$ and $\mu A B^{m-1} \chi\left(C A^{-1} B^{-m}\right) \leqq \omega^{2}$.

By setting $\phi(t)=(T-t)^{-1 /(m-1)}, \phi(t)=\psi(t)$ and $\phi(t)=\int_{0}^{t} \psi^{m}(s) d s$ respectively in Theorem 3 , one obtains the following specific estimates.

Corollary. Let

$$
\begin{gathered}
v=[2 m(m+1) /(m-1)]^{1 / 2}, \\
B=\limsup _{t \uparrow T}(T-t)^{1 /(m-1)} \psi(t), \quad b=\liminf _{t \uparrow T}(T-t)^{1 /(m-1)} \psi(t),
\end{gathered}
$$

and

$$
C=\limsup _{t \uparrow T}(T-t)^{1 /(m-1)} \int_{0}^{t} \psi^{m}(s) d s
$$

Then

$$
v b^{(m-1) / 2} \leqq \omega \leqq \nu B^{(m-1) / 2} .
$$

Moreover, if $0<B<\infty$, then $C \leqq(m-1) B^{m}$ and

$$
v B^{(m-1) / 2} \chi^{1 / 2}\left((m-1)^{-1} C B^{-m}\right) \leqq \omega .
$$


Corollary. Suppose that $\psi \in C^{1}(0, T)$ and let

$$
A=\limsup _{t \uparrow T} \psi^{m}(t) / \psi^{\prime}(t), \quad a=\liminf _{t \uparrow T} \psi^{m}(t) / \psi^{\prime}(t),
$$

and

$$
\varrho=\limsup _{t \uparrow \boldsymbol{T}} \frac{\int_{0}^{t} \psi^{m}(s) d s}{\psi(t)}
$$

Then

$$
\mu a \leqq \omega^{2} \leqq \mu A .
$$

Moreover, if $0<A<\infty$, then $\varrho \leqq A$ and

$$
\mu A \chi\left(\varrho A^{-1}\right) \leqq \omega^{2} .
$$

Corollary. Let $\varrho$ be defined by (1.9) and

$$
\sigma=\liminf _{t \uparrow T}\left[\int_{0}^{t} \psi^{m}(s) d s / \psi(t)\right] .
$$

Then, if $0<\sigma \leqq \varrho<\infty$, there holds

$$
\mu \varrho \max \left\{r^{m}, r^{1-m} \chi\left(r^{m}\right)\right\} \leqq \omega^{2} \leqq \mu \varrho r^{1-m}
$$

where

$$
r=\sigma / \varrho \text {. }
$$

To illustrate the results listed so far, suppose that

$$
\psi(t) \sim \psi_{0}(T-t)^{-q} \text { as } t \uparrow T
$$

for some $\psi_{0}>0$ and $q>0$. Then Theorem 1 states that localization occurs if and only if $q \leqq 1 /(m-1)$. Whilst, it follows from Theorem 2 and the first corollary to Theorem 3 that $\Omega=\{0\}$ if $q<1 /(m-1),\left[0, v \psi_{0}^{(m-1) / 2}\right) \subseteq \Omega \subseteq\left[0, v \psi_{0}^{(m-1) / 2}\right]$ if $q=1 /(m-1)$, and $\Omega=[0, \infty)$ if $q>1 /(m-1)$. These conclusions are borne out by a class of similarity solutions of equation (1.1) of the form: $[5,7,8]$

$$
u(x, t)=(T-t)^{-q} f(\xi), \quad \xi=x(T-t)^{-[1-(m-1) q] / 2} .
$$

Our last result is an estimate of the size of the blow-up set $\Omega$ solely in terms of the factor (1.9) entering into the criterion (1.5) for localization.

Theorem 4. Let $\varrho$ be given by (1.9). Then

$$
m^{-2}(m+1)(2 m+1) \varrho \leqq \omega^{2} \leqq 2(m-1)^{-2} m^{(2 m+1) / m}(m+1)^{1 / m} \varrho .
$$

The relationship between the estimates in Theorems 3 and 4 will be analysed in an appendix to this paper (Appendix A). A corollary of Theorem 4 is that $\Omega=\{0\}$ if and only if $\varrho=0$.

The behaviour of solutions of problem (1.1)-(1.3) with condition (1.4) has been previously studied by Galaktionov et al. [2] and by Galaktionov and Samarkkii [3]. In [2], the first corollary to Theorem 3 was obtained under the specific 
assumption that $\psi(t) \equiv \psi_{0}(T-t)^{-1 /(m-1)}$ for some $\psi_{0}>0$. In [3], it was assumed that in addition to the basic hypotheses the function

$$
\psi \in C^{2}(0, T), \psi^{\prime}(t)>0 \text { for all } t \in(0, T),
$$

and

$$
l=\lim _{t \uparrow T}\left(\psi / \psi^{\prime}\right)^{\prime}(t) \text { exists }
$$

Under these assumptions, Galaktionov and Samarskii have shown that, subject to normalization by $\psi(t)$, the solution $u(x, t)$ converges to an "approximate selfsimilar solution" of problem (1.1)-(1.3) as $t \uparrow T$, uniformly with respect to $x \in[0, \infty)$. The form of the "approximate self-similar solution" infers that localization occurs if and only if

$$
l=-\infty \text { or } \psi^{m} / \psi^{\prime} \in L^{\infty}(0, T)
$$

and that the supremum of the blow-up set satisfies

$$
\omega=\left\{\begin{array}{lll}
0 & \text { if } l=-\infty \\
C \limsup _{t \uparrow r}\left[\psi^{m}(t) / \psi^{\prime}(t)\right]^{1 / 2} & \text { if } \quad l>-\infty,
\end{array}\right.
$$

where $C$ is a positive constant which depends only on $l$. In particular,

$$
C=\mu^{1 / 2} \quad \text { if } \quad l=-(m-1),
$$

cf. (1.6). Our theorems confirm these inferences. In a second appendix to this paper (Appendix B), we discuss the significance of conditions (1.12) and (1.13) and show that under such assumptions

$$
\limsup _{t \uparrow T} \psi^{m}(t) / \psi^{\prime}(t)=\varrho,
$$

where $\varrho$ is defined by (1.9). Furthermore,

$$
\varrho=0 \quad \text { if } \quad l<-(m-1)
$$

and

$$
\varrho=\infty \text { if } l>-(m-1) .
$$

Consequently, under hypotheses (1.12) and (1.13), the criteria for localization (1.5) and (1.14) are equivalent. Moreover, in the light of the second corollary to Theorem 3 and of Theorem 4,(1.15) is a true estimate of the size of the blow-up set which can be formulated as

$$
\omega=\mu^{1 / 2} \varrho^{1 / 2} \text { irrespective of the value of } l \text {. }
$$

When $m=1$, problem (1.1)-(1.3) can be solved explicitly. For instance, if $u_{0} \equiv 0$ one has

$$
u(x, t)=\frac{x}{2 \sqrt{\pi}} \int_{0}^{t} \exp \left[-\frac{x^{2}}{4(t-s)}\right](t-s)^{-3 / 2} \psi(s) d s
$$


Note that thermal fronts are absent in this case. It is however said that metastable localization occurs if there exists an $x>0$ such that $\int_{x}^{\infty} u(y, t) d y$ remains bounded as $t \uparrow T[16]$. Let $x_{f}$ denote the infimum of the set of such points $x$. Then, supposing that

$$
\psi(t) \sim(T-t)^{p} \exp \left[\alpha(T-t)^{-q}\right] \quad \text { as } t \uparrow T
$$

for some $p, \alpha>0$, and $q>0$, the following has been remarked in [16]. When $q<1$, one has metastable localization with $x_{f}=0$. When $q=1$, metastable localization occurs with $x_{f}=2 \alpha^{1 / 2}$. Moreover, $u(x, t) \uparrow \infty$ as $t \uparrow T$ for all $x<x_{f}$, and $u\left(x_{f}, t\right) \uparrow \infty$ as $t \uparrow T$ if $p \leqq 1 / 2$. Finally, when $q>1, u(x, t) \uparrow \infty$ as $t \uparrow T$ for all $x$. These cases are called the LS, S, and HS regimes, respectively. Actually, in both the above cases of metastable localization the temperature remains bounded by the limiting distribution

$$
\bar{u}(x, t)=\frac{x^{2 p}}{4^{p} \sqrt{\pi}} \int_{x^{2} / 4 T}^{\infty} \exp \left[-z+\alpha\left(\frac{4 z}{x^{2}}\right)^{q}\right] z^{-p-1 / 2} d z
$$

as can be deduced from (1.16).

The remainder of this paper is organized as follows. In the next section, we review some useful properties of solutions of problem (1.1)-(1.3). Thereafter, we prove each of the four theorems in turn in a separate section. As appendices we discuss the relationship between Theorems 3 and 4, and the connection between our results and the work of Galaktionov and Samarskii [3] under assumptions (1.12) and (1.13).

\section{Preliminaries}

Here we state precisely what is meant by a generalized solution of problem (1.1)-(1.3), and recall for convenience some basic properties of such a solution. We let

$$
H=(0, \infty) \times(0, T)
$$

A function $u(x, t)$ is said to be a generalized supersolution of equation (1.1) in a domain

$$
D=\left(\eta_{1}, \eta_{2}\right) \times\left(\tau_{1}, \tau_{2}\right]
$$

with

$$
0 \leqq \eta_{1}<\eta_{2} \leqq \infty \text { and } 0 \leqq \tau_{1}<\tau_{2}<T,
$$

if $u$ is defined, bounded, nonnegative and continuous in $\bar{D}$ and satisfies the integral inequality

$$
\begin{aligned}
& \int_{t_{1}}^{t_{2}} \int_{x_{1}}^{x_{2}}\left\{u^{m} \theta_{x x}+u \theta_{t}\right\} d x d t \\
& \leqq \int_{x_{1}}^{x_{2}}\left\{u\left(x, t_{2}\right) \theta\left(x, t_{2}\right)-u\left(x, t_{1}\right) \theta\left(x, t_{1}\right)\right\} d x \\
& \quad+\int_{t_{1}}^{t_{2}}\left\{u^{m}\left(x_{2}, t\right) \theta_{x}\left(x_{2}, t\right)-u^{m}\left(x_{1}, t\right) \theta_{x}\left(x_{1}, t\right)\right\} d t
\end{aligned}
$$


for all non-empty bounded rectangles $R=\left(x_{1}, x_{2}\right) \times\left(t_{1}, t_{2}\right] \subseteq D$ and nonnegative functions $\theta \in C^{2,1}(\bar{R})$ such that $\theta\left(x_{1}, t\right)=\theta\left(x_{2}, t\right)=0$ for all $t \in\left[t_{1}, t_{2}\right]$. Similarly, a function is said to be a generalized subsolution of equation (1.1) in $D$ if it satisfies the above criteria with the inequality in $(2.3)$ reversed. A function $u(x, t)$ is then said to be a generalized solution of problem (1.1)-(1.3) if it is a generalized supersolution and a generalized subsolution of equation $(1.1)$ in $(0, \infty) \times(0, \tau]$ for all $\tau \in(0, T)$, and satisfies (1.2) and (1.3).

Under the hypotheses that $u_{0}$ is bounded, nonnegative, and continuous on $[0, \infty), \psi(t)$ is nonnegative and continuous on $[0, T)$, and $u_{0}$ and $\psi$ satisfy the compatibility condition $\boldsymbol{u}_{0}(0)=\psi(0)$, problem $(1.1)-(1.3)$ has a unique generalized solution $u(x, t)$ which is a smooth classical solution of equation (1.1) in a neighbourhood of any point $(x, t) \in H$ where $u(x, t)>0$. However $u$ may fail to be a classical solution at points where $u(x, t)=0[6,13]$.

In contrast to the linear problem with $m=1$, in the present case of $m>1$, if $u_{0}$ has compact support, then the generalized solution $u(x, t)$ of problem (1.1)-(1.3) also has compact support as a function of $x$ for all $t \in(0, T)$. Thus the interface

$$
\zeta(t)=\sup \{x \in[0, \infty): u(x, t)>0\}
$$

is well-defined. Furthermore, it is to be noted that $\zeta(t)$ is continuous and monotonic increasing on $[0, T)[9,13]$.

The following basic properties of generalized solutions will be used in this analysis.

Lemma 1. (Comparison Principle) $[6,13]$. Let $u(x, t)$ denote a generalized solution of problem (1.1)-(1.3) and $D$ a domain of the form (2.1), (2.2).

(a) If $U(x, t)$ is a generalized supersolution of equation (1.1) in D such that $U(x, t)$ $\geqq u(x, t)$ for all $(x, t) \in \bar{D} \backslash D$ there holds $U(x, t) \geqq u(x, t)$ for all $(x, t) \in \bar{D}$.

(b) If $U(x, t)$ is a generalized subsolution of equation (1.1) in $D$ such that $U(x, t)$ $\leqq u(x, t)$ for all $(x, t) \in \bar{D} \backslash D$ there holds $U(x, t) \leqq u(x, t)$ for all $(x, t) \in \bar{D}$.

Lemma 2. Let $u(x, t)$ denote a generalized solution of problem (1.1)-(1.3) and suppose that $\psi(t)$ is monotonic increasing. Set

$$
M_{0}=\sup \left\{u_{0}(x): x \in[0, \infty)\right\} .
$$

Then $u(x, t) \leqq \max \left\{M_{0}, \psi(t)\right\}$ for all $(x, t) \in H$.

Proof. Fix $t_{1} \in[0, T)$ and write $C=\max \left\{M_{0}, \psi\left(t_{1}\right)\right\}$. Since $\psi(t)$ is monotonic increasing, $\psi(t) \leqq C$ for all $t \in\left[0, t_{1}\right]$. Moreover, by definition, $u_{0}(x) \leqq C$ for all $x \in[0, \infty)$. As $C$ itself is a generalized solution of $(1.1)-(1.3)$ with the obvious initial and boundary values, it follows from Lemma 1 that $u(x, t) \leqq C$ whenever $(x, t) \in[0, \infty) \times\left[0, t_{1}\right]$. Whence the conclusion, since $t_{1}<T$ was arbitrary.

Lemma 3. Suppose that the hypotheses of Lemma 2 are satisfied. Then, if $u_{0}$ has compact support, given any point $\left(x_{1}, t_{1}\right) \in H$ such that $u\left(x_{1}, t_{1}\right)>M_{0}$ there holds $u\left(x, t_{1}\right) \geqq u\left(x_{1}, t_{1}\right)$ for all $x \in\left[0, x_{1}\right)$.

Proof. Assume the result to be false, so that there exists a point $x_{0} \in\left[0, x_{1}\right)$ such that $m_{0}=u\left(x_{0}, t_{1}\right)<m_{1}=u\left(x_{1}, t_{1}\right)$. In view of the continuity of $u$, without any loss of 
generality we may suppose that $m_{0}>M_{0}$ and that $\left(x_{0}, t_{1}\right)$ and $\left(x_{1}, t_{1}\right)$ belong to the same connected component $G$ of the set $\{(x, t) \in H: u(x, t)>0\}$. Set $C=\left(m_{0}+m_{1}\right) / 2$. Then $C$ is certainly a classical solution of equation (1.1) in $G$. Moreover, $u(x, t)-C$ changes sign at least twice on $\left\{\left(x, t_{1}\right) \in G\right\}$. By a standard argument $[19,20]$, it follows that $u(x, t)-C$ must subsequently change sign at least twice on the parabolic boundary of $G$. And, because $C>M_{0} \geqq 0$, this can only be achieved along the line $\{0\} \times(0, T)$. But, this is impossible, since $\psi(t)$ is monotonic.

We conclude this section with the principal tool in the ensuing analysis.

Lemma 4 [4]. Let $u(x, t)$ denote a generalized solution of problem (1.1)-(1.3) and suppose that $u_{0}$ has compact support. Then

$$
\int_{0}^{\infty} x u(x, t) d x=\int_{0}^{\infty} x u_{0}(x) d x+\int_{0}^{t} \psi^{m}(s) d s
$$

for all $t \in[0, T)$.

To formally derive (2.5) it suffices to multiply (1.1) by $x$ and integrate by parts.

\section{The Proof of Theorem 1}

We begin with the proof of the necessity of (1.5).

Let $M_{0}$ be given by (2.4), and $\tau_{0}=\sup \left\{t \in[0, T): \psi(t) \leqq M_{0}\right\}$. By Lemmata 2 and 4 , for any $t \in\left[\tau_{0}, T\right)$ one has subsequently

$$
\int_{0}^{t} \psi^{m}(s) d s \leqq \int_{0}^{\zeta(t)} x u(x, t) d x \leqq \frac{1}{2} \psi(t) \zeta^{2}(t)
$$

Whence, if localization occurs, (1.5) holds.

To show the sufficiency of (1.5) for localization, we proceed in several steps, beginning with the following estimate.

Lemma 5. Let $\varrho$ be given by (1.9), then

$$
\underset{t \uparrow T}{\limsup }(T-t)^{1 /(m-1)} \int_{0}^{t} \psi^{m}(s) d s \leqq(m-1)^{-1 /(m-1)} \varrho^{m /(m-1)} .
$$

Proof. If $\psi \in L^{m}(0, T)$ or $\varrho=\infty,(3.1)$ holds trivially. We may therefore suppose that

$$
\int_{0}^{t} \psi^{m}(s) d s \uparrow \infty \quad \text { as } \quad t \uparrow T
$$

and $\varrho<\infty$. Then, given any $\varepsilon>0$, there exists a $\tau \in[0, T)$ such that

$$
0<\int_{0}^{t} \psi^{m}(s) d s<(\varrho+\varepsilon) \psi(t) \text { for all } t \in[\tau, T) .
$$

We rewrite this as

$$
(m-1)(\varrho+\varepsilon)^{-m}<-\frac{d}{d t}\left[\int_{0}^{t} \psi^{m}(s) d s\right]^{1-m} .
$$


Whence, integrating from $t_{1} \in[\tau, T)$ to $t_{2} \in\left(t_{1}, T\right)$,

$$
(m-1)(\varrho+\varepsilon)^{-m}\left(t_{2}-t_{1}\right)<\left[\int_{0}^{t_{1}} \psi^{m}(s) d s\right]^{1-m}-\left[\int_{0}^{t_{2}} \psi^{m}(s) d s\right]^{1-m} .
$$

We now let $t_{2} \uparrow T$ and use (3.2) to deduce

$$
(m-1)(\varrho+\varepsilon)^{-m}\left(T-t_{1}\right) \leqq\left[\int_{0}^{t_{1}} \psi^{m}(s) d s\right]^{1-m},
$$

from which, since $t_{1} \in[\tau, T)$ was arbitrary, (3.1) with $\varrho$ replaced by $(\varrho+\varepsilon)$ follows. To wrap up, we may let $\varepsilon \downarrow 0$.

Lemma 6. Let $\varrho$ be given by (1.9), then

$$
\limsup _{t \uparrow T}(T-t)^{1 /(m-1)} u(x, t) \leqq 2(m-1)^{-1 /(m-1)} Q^{m /(m-1)} x^{-2}
$$

for all $x>0$.

Proof. Pick $x>0$ but otherwise arbitrary. Let

$$
\sigma=\limsup _{t \uparrow T}(T-t)^{1 /(m-1)} u(x, t),
$$

and let $\left\{t_{i}\right\}_{i=1}^{\infty}$ denote an increasing sequence of values in $(0, T)$ such that

$$
t_{i} \uparrow T \text { and }\left(T-t_{i}\right)^{1 /(m-1)} u\left(x, t_{i}\right) \rightarrow \sigma \quad \text { as } i \uparrow \infty .
$$

Without any loss of generality, recalling the definition of $M_{0}$ in (2.4), we may suppose that $u\left(x, t_{i}\right)>M_{0}$ for all $i \geqq 1$, so that, by Lemma $3, u\left(y, t_{i}\right) \geqq u\left(x, t_{i}\right)$ for all $y \in[0, x]$ and $i \geqq 1$. Whence

$$
\frac{1}{2} x^{2} u\left(x, t_{i}\right) \leqq \int_{0}^{x} y u\left(y, t_{i}\right) d y \leqq \int_{0}^{\infty} y u\left(y, t_{i}\right) d y
$$

for all $i \geqq 1$. On the other hand, by Lemma 4 ,

$$
\int_{0}^{\infty} y u\left(y, t_{i}\right) d y=\int_{0}^{\infty} y u_{0}(y) d y+\int_{0}^{t_{i}} \psi^{m}(s) d s
$$

Using (3.1), (3.3) is immediate.

Lemma 7. Suppose that localization does not occur. Then for any $x \in[0, \infty)$,

$$
\underset{t \uparrow T}{\lim \sup }(T-t)^{1 /(m-1)} u(x, t)=\infty .
$$

Proof. We argue by contradiction. Thus assume that there are nonnegative real numbers $x_{0}$ and $B$ such that

$$
(T-t)^{1 /(m-1)} u\left(x_{0}, t\right) \leqq B<\infty \text { for all } t \in[0, T) .
$$

Consider the function

$$
U(x, t ; c)= \begin{cases}{\left[\frac{m-1}{2 m(m+1)} \frac{(c-x)^{2}}{T-t}\right]^{1 /(m-1)}} & \text { if } x<c \\ 0 & \text { if } x \geqq c .\end{cases}
$$


For any $c, U(x, t ; c)$ is an explicit generalized solution of problem (1.1)-(1.3) with appropriate initial and boundary conditions [9]. Select now the constant $c>0$ so large that

$$
(T-t)^{1 /(m-1)} U\left(x_{0}, t ; c\right) \geqq B \text { for all } t \in[0, T)
$$

and

$$
U(x, 0 ; c) \geqq u_{0}(x) \text { for all } x \in[0, \infty) .
$$

These conditions are met, for instance, if

$$
c>\max \left\{\zeta(0)+v M_{0}^{(m-1) / 2} T^{1 / 2}, x_{0}+v B^{(m-1) / 2}\right\},
$$

where $v$ is defined by (1.8) and $M_{0}$ by (2.4). Subsequently, comparing $u(x, t)$ and $U(x, t ; c)$ in the region $\left(x_{0}, \infty\right) \times(0, T)$, and using (3.4)-(3.6), by Lemma 1 , one must conclude that

$$
u(x, t) \leqq U(x, t ; c) \text { for all }(x, t) \in\left[x_{0}, \infty\right) \times[0, T) .
$$

However, this implies that $\zeta(t) \leqq c$ for all $t \in[0, T)$. So localization occurs. This contradiction completes the proof of the lemma.

Plainly, combining Lemmata 6 and 7 establishes the sufficiency of criterion (1.5) for localization, and therewith the remaining assertions of Theorem 1.

\section{The Proof of Theorem 2}

Theorem 2 is an immediate corollary of the following result and Lemma 3.

Lemma 8. Let $u(x, t)$ denote a generalized solution of problem (1.1)-(1.3) and suppose that $\psi(t)$ is monotonic increasing and that $u_{0}$ has compact support. Then given any point $\left(x_{1}, t_{1}\right) \in H$ there holds

$$
u\left(x_{1}, t\right) \geqq u\left(x_{1}, t_{1}\right)\left[t_{1} / t\right]^{1 /(m-1)} \text { for all } t \in\left[t_{1}, T\right) .
$$

Proof. Pick arbitrary $\tau \in(0, T)$. Then in view of the properties of the interface, $\zeta(t)$, there exists an $\eta \in(0, \infty)$ such that $u(x, t)=0$ for all $x \geqq \eta$ and $t \in[0, \tau]$. Set

$$
R=(0, \eta) \times(0, \tau] \text {. }
$$

Now, in $R, u$ may be regarded as a generalized solution of the first boundary-value problem for equation (1.1) with data:

$$
\begin{array}{lll}
u(0, t)=\psi(t) & \text { for } & 0<t \leqq \tau, \\
u(x, 0)=u_{0}(x) & \text { for } & 0 \leqq x \leqq \eta, \\
u(\eta, t)=0 & \text { for } & 0<t \leqq \tau .
\end{array}
$$

Subsequently [6], in $\bar{R}, u$ may be constructed as the pointwise limit of a sequence of functions $\left\{u_{k}\right\}_{k=1}^{\infty}$ with the properties:

(i) $u_{k} \in C^{2,1}(\bar{R}) \cap C^{\infty}(R)$;

(ii) there exists an $\varepsilon_{k}>0$ such that $u_{k}(x, t) \geqq \varepsilon_{k}$ for all $(x, t) \in \bar{R}$;

(iii) $u_{k}$ is a classical solution of equation (1.1) in $\bar{R}$;

(iv) $u_{k} \downarrow u$ as $k \uparrow \infty$ uniformly on $\bar{R}$;

(v) $\frac{\partial}{\partial t} u_{k}(0, t) \geqq 0$ and $\frac{\partial}{\partial t} u_{k}(\eta, t)=0$ for all $t \in[0, T]$. 
Consider now the function $z=u_{k}+(m-1) t \frac{\partial}{\partial t} u_{k}$. There holds

$$
z_{t}=\left(m u_{k}^{m-1} z\right)_{x x} \text { in } R \text {. }
$$

Cf. [1]. However, in view of property (v) above, $z(x, t) \geqq u_{k}(x, t) \geqq 0$ for all $(x, t) \in \bar{R} \backslash R$. Hence, by the classic maximum principle for parabolic equations, $z(x, t) \geqq 0$ for all $(x, t) \in \bar{R}$. Integrating subsequently yields $u_{k}\left(x_{1}, t\right)$ $\geqq u_{k}\left(x_{1}, t_{1}\right)\left[t_{1} / t\right]^{1 /(m-1)}$ for all $\left(x_{1}, t_{1}\right) \in \bar{R}$ and $t \in\left[t_{1}, \tau\right]$. The lemma finally ensues by taking the successive limits $k \uparrow \infty, \eta \uparrow \infty$, and $\tau \uparrow T$.

In the terminology of $[10,11]$, Lemma 3 says that the blow-up set $\Omega$ associated with problem (1.1)-(1.3) is a simple structure.

\section{The Proof of Theorem 3}

To prove Theorem 3 , the following auxiliary result will be used.

Lemma 9. Let $u_{1}$ and $u_{2}$ denote two generalized solutions of problem(1.1)-(1.3) with boundary data $u_{0,1}, \psi_{1}$ and $u_{0,2}, \psi_{2}$ respectively. Let $\omega_{1}$ and $\omega_{2}$ denote the corresponding suprema of their blow-up sets. Then if there exists a $\tau \in[0, T)$ such that $\psi_{1}(t) \leqq \psi_{2}(t)$ for all $t \in[\tau, T)$ there holds $\omega_{1} \leqq \omega_{2}$.

Proof. We expand on an idea in [2]. Define

$$
\psi^{*}(t)=\max \left\{\psi_{1}(t), \psi_{2}(t)\right\} \text { for } t \in[0, T)
$$

and

$$
u_{0}^{*}(x)=\max \left\{u_{0,1}(x), u_{0,2}(x)\right\} \quad \text { for } \quad x \in[0, \infty) .
$$

Let $u^{*}$ denote the solution of problem (1.1)-(1.3) with data $u_{0}^{*}, \psi^{*}$, and let $\omega^{*}$ denote the supremum of the corresponding blow-up set. By Lemma 1,

$$
u_{1}(x, t) \leqq u^{*}(x, t) \text { and } u_{2}(x, t) \leqq u^{*}(x, t) \text { for all }(x, t) \in H .
$$

Hence, $\omega_{1} \leqq \omega^{*}$ and $\omega_{2} \leqq \omega^{*}$. Accordingly, we shall achieve our goal if we prove that $\omega_{2}=\omega^{*}$. By Lemma 4,

$$
\begin{aligned}
\int_{0}^{\infty} x & {\left[u^{*}(x, t)-u_{2}(x, t)\right] d x } \\
& =\int_{0}^{\infty} x\left[u_{0}^{*}(x)-u_{0,2}(x)\right] d x+\int_{0}^{t}\left[\left(\psi^{*}\right)^{m}(s)-\left(\psi_{2}\right)^{m}(s)\right] d s
\end{aligned}
$$

for all $t \in[0, T)$. Subsequently, by (5.1) and the definition of $\psi^{*}$,

$$
\begin{aligned}
\int_{0}^{\infty} x & {\left[u^{*}(x, t)-u_{2}(x, t)\right] d x } \\
& =\int_{0}^{\infty} x\left[u_{0}^{*}(x)-u_{0,2}(x)\right] d x+\int_{0}^{\tau}\left[\left(\psi^{*}\right)^{m}(s)-\left(\psi_{2}\right)^{m}(s)\right] d s \\
& =C_{0}<\infty
\end{aligned}
$$

for all $t \in[\tau, T)$. Suppose now that $\omega_{2}<\omega^{*}$. Then, in view of (5.1) and (5.2), we can pick points $x_{a} \in\left(\omega_{2}, \omega^{*}\right)$ and $x_{b} \in\left(x_{a}, \omega^{*}\right)$ such that

$$
0 \leqq \int_{x_{a}}^{x_{b}} x\left[u^{*}(x, t)-u_{2}(x, t)\right] d x \leqq C_{0}
$$


for all $t \in[\tau, T)$. However, since $x_{a}>\omega_{2}$, by Lemmata 1 and 2 ,

$$
\begin{aligned}
& \int_{x_{a}}^{x_{b}} x u_{2}(x, t) d x \leqq \frac{1}{2}\left(x_{b}^{2}-x_{a}^{2}\right) \\
& \quad \times \max \left\{\sup \left\{u_{2}(x, \tau): x \in\left[x_{a}, \infty\right)\right\}, \sup \left\{u_{2}\left(x_{a}, t\right): t \in[\tau, T)\right\}\right\} \\
& =C_{1}<\infty
\end{aligned}
$$

for all $t \in[\tau, T)$. On the other hand, since $x_{b}<\omega^{*}$, there exists a sequence of values $\left\{t_{i}\right\}_{i=1}^{\infty}$ such that $t_{i} \uparrow T$ and

$$
u^{*}\left(x_{b}, t_{i}\right) \uparrow \infty \text { as } i \uparrow \infty .
$$

Without any loss of generality, we may assume that $u^{*}\left(x_{b}, t_{i}\right)$ $>\sup \left\{u_{0}^{*}(x): x \in[0, \infty)\right\}$ for all $i \geqq 1$. But then Lemma 3 implies that $u^{*}\left(x, t_{i}\right) \uparrow \infty$ as $i \uparrow \infty$ uniformly on $\left[x_{a}, x_{b}\right]$. Thus, in (5.3), we have a contradiction between (5.4) and (5.5).

Let us now turn to the proof of part (i) of the theorem.

Fix $\varepsilon>0$, and choose $\tau \in(0, T)$ such that

$$
\phi^{m}(t) \leqq(A+\varepsilon) \phi^{\prime}(t) \text { and } 0<\psi(t) \leqq(B+\varepsilon) \phi(t) \text { for all } t \in[\tau, T) .
$$

Consider the function

$$
v(x, t)=(B+\varepsilon) \phi(t)\left[1-x / p_{\varepsilon}\right]_{+}^{2 /(m-1)},
$$

where $[y]_{+}=\max \{0, y\}$ and

$$
p_{\varepsilon}=\left[\mu(A+\varepsilon)(B+\varepsilon)^{m-1}\right]^{1 / 2} .
$$

It is easily verified that $v$ is a classical supersolution and hence also a generalized supersolution of equation (1.1) in subsets of $(0, \infty) \times(\tau, T)$ of the form $(2.1),(2.2)$. Hence, if we denote by $u_{\varepsilon}(x, t)$ the generalized solution of equation $(1.1)$ in $(0, \infty)$ $\times(\tau, T)$ with data

$$
u_{\varepsilon}(x, \tau)=\min \{u(x, \tau), v(x, \tau)\} \text { for } 0 \leqq x<\infty
$$

and

$$
u_{\varepsilon}(0, t)=\psi(t) \quad \text { for } \quad \tau \leqq t<T
$$

by Lemma 1 , there holds

$$
u_{\varepsilon}(x, t) \leqq v(x, t) \text { for all }(x, t) \in[0, \infty) \times[\tau, T) .
$$

This means that the supremum of the blow-up set of $u_{\varepsilon}$ is less than or equal to $p_{\varepsilon}$ However, by Lemma 9 , the supremum of the blow-up set of $u_{\varepsilon}$ is identical to $\omega$. So

$$
\omega \leqq p_{\varepsilon} .
$$

Since $\varepsilon>0$ was arbitrary this verifies part (i) of the theorem.

The proof of part (ii) of Theorem 3 is analogous to the proof of part (i); so we omit the details. To prove part (iii), we extend the preceeding analysis.

By Lemma 4,

$$
\int_{\tau}^{t} \psi^{m}(s) d s=\int_{0}^{\infty} x u_{\varepsilon}(x, t) d x-\int_{0}^{\infty} x u_{\varepsilon}(x, \tau) d x
$$


for all $t \in(\tau, T)$. Therefore, using (5.7),

$$
\int_{0}^{t} \psi^{m}(s) d s \leqq \int_{0}^{\infty} x v(x, t) d x+\int_{\infty}^{\infty} x u_{\varepsilon}(x, t) d x+\int_{0}^{\tau} \psi^{m}(s) d s
$$

and consequently, by (5.6),

$$
\begin{aligned}
\int_{0}^{t} \psi^{m}(s) d s / \phi(t) \leqq & \int_{0}^{\infty} x v(x, t) / \phi(t) d x \\
& +(B+\varepsilon)\left[\int_{\omega}^{\infty} x u_{\varepsilon}(x, t) d x+\int_{0}^{t} \psi^{m}(s) d s\right] / \psi(t),
\end{aligned}
$$

for all $t \in(\tau, T)$. Letting $t \uparrow T$ in an appropriate fashion and applying Fatou's Lemma we subsequently deduce

$$
C \leqq \int_{0}^{\omega} x(B+\varepsilon)\left[1-x / p_{\varepsilon}\right]_{+}^{2 /(m-1)} d x .
$$

Changing variables though, this last statement is equivalent to

$$
C(B+\varepsilon)^{-m}(A+\varepsilon)^{-1} \leqq \frac{1}{2} \mu \int_{0}^{\omega^{2} / p_{\varepsilon}^{2}}\left(1-\xi^{1 / 2}\right)^{2 /(m-1)} d \xi .
$$

Whence, in the limit $\varepsilon \downarrow 0$, we obtain the desired result.

\section{The Proof of Theorem 4}

If $\varrho=\infty$ then localization is absent, and the theorem is covered by the preceeding results. To prove Theorem 4 it therefore suffices to verify (1.11) in the event that $\varrho<\infty$, which, in view of Theorems 1 and 2 , is the case if and only if $\omega<\infty$.

We enlist the next result.

Lemma 10. Let $u(x, t)$ denote a generalized solution of problem (1.1)-(1.3) and suppose that $\psi(t)$ is monotonic increasing and that $u_{0} \equiv 0$. Then given any $t \in[0, T)$, $u^{m}(x, t)$ is a convex function of $x$ on $[0, \infty)$.

Proof. We take up the proof of Lemma 8 . If $u_{0} \equiv 0$, then we may add the following to the list of properties of the approximating sequence of functions $\left\{u_{k}\right\}_{k=1}^{\infty}$ :

$$
\text { (vi) } \frac{\partial}{\partial x} u_{k}(x, 0)=0 \text { for all } x \in[0, \eta] \text {. }
$$

Subsequently, considering $z=\frac{\partial}{\partial t} u_{k}=\frac{\partial^{2}}{\partial x^{2}} u_{k}^{m}$ in $\widetilde{R}$, one can verify that it satisfies (4.1) and also $z(x, t) \geqq 0$ for all $(x, t) \in \bar{R} \backslash R$. Whence, by the standard maximum principle for nonlinear parabolic equations, one can deduce that $z(x, t) \geqq 0$ for all $(x, t) \in \bar{R}$. Consequently, integration yields

$$
u_{k}^{m}\left(x_{2}, t\right) \leqq\left[u_{k}^{m}\left(x_{3}, t\right)\left(x_{2}-x_{1}\right)+u_{k}^{m}\left(x_{1}, t\right)\left(x_{3}-x_{2}\right)\right] /\left(x_{3}-x_{1}\right)
$$

for all $t \in[0, \tau], x_{1} \in[0, \eta), x_{2} \in\left(x_{1}, \eta\right)$, and $x_{3} \in\left(x_{2}, \eta\right]$. The desired result follows by taking the limits $k \uparrow \infty, \eta \uparrow \infty$, and $\tau \uparrow T$, as in the completion of the proof of Lemma 8.

An impugnable alternative proof of Lemma 10 can be found in [12]. 
We are now in a position to confirm the left-hand inequality in (1.11). In view of Lemma 9 , without any loss of generality, we may suppose that $u_{0} \equiv 0$. In which case, by Lemma 10 , given any $\eta \in(0, \infty)$ there holds

$$
u(x, t) \leqq\left\{\left[u^{m}(\eta, t) x+\psi^{m}(t)(\eta-x)\right] / \eta\right\}^{1 / m}
$$

for all $x \in[0, \eta]$. Substituting this inequality into (2.5), and dividing by $\psi(t)$ subsequently yields

$$
\begin{aligned}
& \int_{0}^{t} \psi^{m}(s) d s / \psi(t) \\
& \quad \leqq \int_{0}^{\eta} x\left\{\frac{u^{m}(\eta, t) x+\psi^{m}(t)(\eta-x)}{\eta \psi^{m}(t)}\right\}^{1 / m} d x+\int_{\eta}^{\zeta(t)} x u(x, t) / \psi(t) d x
\end{aligned}
$$

for all $t \in(0, T)$. Supposing now $\eta>\omega$, letting $t \uparrow T$ and using the Dominated Convergence Theorem, we deduce

$$
\varrho \leqq \int_{0}^{\eta} x\{(\eta-x) / \eta\}^{1 / m} d x=m^{2}(m+1)^{-1}(2 m+1)^{-1} \eta^{2} .
$$

Letting $\eta \downarrow \omega$, the result is immediate.

As to the right-hand inequality in (1.11), Lemma 6 states that

$$
\limsup _{t \uparrow T}(T-t)^{1 /(m-1)} u(\eta, t) \leqq 2(m-1)^{-1 /(m-1)} \varrho^{m /(m-1)} \eta^{-2}
$$

for all $\eta \in(0, \infty)$. Consequently, applying the first corollary of Theorem 3 to the generalized solution of problem (1.1)-(1.3) $\tilde{u}(x, t)=u(x+\eta, t)$ with appropriately adapted boundary data, and taking (6.1) into account, we obtain

$$
\begin{aligned}
\omega & \leqq \eta+v\left\{2(m-1)^{-1 /(m-1)} \varrho^{m /(m-1)} \eta^{-2}\right\}^{(m-1) / 2} \\
& =\eta+v\left\{2^{m-1}(m-1)^{-1} \varrho^{m}\right\}^{1 / 2} \eta^{1-m},
\end{aligned}
$$

where $v$ is given by (1.8). The right-hand inequality of (1.11) is subsequently derivable from the calculus result below whose proof is elementary and consequentially omitted.

Lemma 11. Suppose that there exists a constant $C \geqq 0$ such that

$$
\omega \leqq \eta+C \eta^{1-m} \text { for all } \eta \in(0, \infty) \text {. }
$$

Then

$$
\omega \leqq m(m-1)^{(1-m) / m} C^{1 / m}
$$

\section{Appendix A. Relation Between Theorems 3 and 4}

The purpose of this appendix is to show that the estimates on the size of the blowup set $\Omega$ obtained in Theorems 3 and 4 are compatible.

The next lemma will be useful. 
Lemma A1. Suppose that the introductory hypotheses of Theorem 3 hold. Then, if $\phi(t) \uparrow \infty$ as $t \uparrow T$,

$$
A \geqq \limsup _{t \uparrow T}\left[\int_{0}^{t} \phi^{m}(s) d s / \phi(t)\right] \geqq \liminf _{t \uparrow T}\left[\int_{0}^{t} \phi^{m}(s) d s / \phi(t)\right] \geqq a
$$

and

$$
(m-1)^{-1} A \geqq \limsup _{t \uparrow T}(T-t) \phi^{m-1}(t) .
$$

Proof. Let us begin with (A.1). Without loss of generality, we may assume that $A<\infty$. Fix $\varepsilon>0$. Then there exists a $\tau \in(0, T)$ such that

$$
\phi^{m}(t) \leqq(A+\varepsilon) \phi^{\prime}(t) \text { for all } t \in[\tau, T) .
$$

Hence, integrating,

$$
\int_{\tau}^{t} \phi^{m}(s) d s \leqq(A+\varepsilon) \phi(t) \text { for all } t \in[\tau, T)
$$

Whence

$$
\int_{0}^{t} \phi^{m}(s) d s / \phi(t) \leqq A+\varepsilon+\int_{0}^{\tau} \phi^{m}(s) d s / \phi(t) \text { for all } t \in[\tau, T) .
$$

Letting $t \uparrow T$ and thereafter $\varepsilon \downarrow 0$, yields the left-hand inequality in (A.1). One establishes the lower bound similarly.

As to (A.2), we write (A.3) as

$$
(m-1) \leqq-(A+\varepsilon)\left(\phi^{1-m}\right)^{\prime}(t) \text { for all } t \in[\tau, T) .
$$

Integrating from $t \in[\tau, T)$ to $T$ subsequently gives

$$
(m-1)(T-t) \leqq(A+\varepsilon) \phi^{1-m}(t) \text { for all } t \in(\tau, T),
$$

which in view of the arbitrariness of $\varepsilon$ confirms the assertion.

Now we come to the crux of the matter.

Lemma A2. Suppose that the hypotheses of Theorem 3 (i) hold and let $\varrho$ be given by (1.9). Then

$$
\varrho \leqq m(m-1)^{-1} A B^{m-1} .
$$

Proof. Fix $\varepsilon>0$. Then there exists a $\tau \in(0, T)$ such that

and

$$
0<\psi(t) \leqq(B+\varepsilon) \phi(t) \text { for all } t \in[\tau, T)
$$

$$
\phi^{m}(t) \leqq(A+\varepsilon) \phi^{\prime}(t) \text { for all } t \in[\tau, T) .
$$

Recalling (1.4), pick $\tau^{*} \in[\tau, T)$ such that $\psi\left(\tau^{*}\right) \geqq(B+\varepsilon) \phi(\tau)$. From (1.4), (A.5), and (A.6), we have $\phi^{\prime}(t)>0$ for all $t \in[\tau, T)$ and $\phi(t) \uparrow \infty$ as $t \uparrow T$. Hence, for $t \in\left[\tau^{*}, T\right)$ we may define $R(t) \in[\tau, T)$ by

$$
(B+\varepsilon) \phi(R(t))=\psi(t)
$$


Moreover, $R(t)$ is a monotonic increasing function of $t \in\left[\tau^{*}, T\right), R(t) \uparrow T$ as $t \uparrow T$, and, in view of (A.5), $R(t) \leqq t$ for all $t \in\left[\tau^{*}, T\right)$. This implies

$$
\begin{aligned}
\int_{0}^{t} \psi^{m}(s) d s & =\int_{0}^{\tau} \psi^{m}(s) d s+\int_{\tau}^{R(t)} \psi^{m}(s) d s+\int_{R(t)}^{t} \psi^{m}(s) d s \\
& \leqq \int_{0}^{\tau} \psi^{m}(s) d s+(B+\varepsilon)^{m} \int_{\tau}^{R(t)} \phi^{m}(s) d s+\int_{R(t)}^{t} \psi^{m}(t) d s \\
& \leqq \int_{0}^{\tau} \psi^{m}(s) d s+(B+\varepsilon)^{m} \int_{0}^{R(t)} \phi^{m}(s) d s+(T-R(t)) \psi^{m}(t)
\end{aligned}
$$

for all $t \in\left[\tau^{*}, T\right)$. So that, using (A.7),

$$
\begin{aligned}
\int_{0}^{t} \psi^{m}(s) d s / \psi(t) \leqq & \int_{0}^{\tau} \psi^{m}(s) d s / \psi(t)+(B+\varepsilon)^{m-1} \int_{0}^{R(t)} \phi^{m}(s) d s / \phi(R(t)) \\
& +(T-R(t))(B+\varepsilon)^{m-1} \phi^{m-1}(R(t))
\end{aligned}
$$

for all $t \in\left[\tau^{*}, T\right)$. Letting $t \uparrow T$ and applying Lemma A1 subsequently yields

$$
\varrho \leqq(B+\varepsilon)^{m-1} A+(B+\varepsilon)^{m-1}(m-1)^{-1} A .
$$

Passing to the limit $\varepsilon \downarrow 0$, gives (A.4).

Lemma A3. Suppose that the hypotheses of Theorem 3 (ii) hold and let $\varrho$ be given by (1.9). Then

$$
a b^{m-1} \leqq \varrho .
$$

Proof. Take any $\varepsilon \in(0, b)$. There exists a $\tau \in(0, T)$ such that

$$
\psi(t) \geqq(b-\varepsilon) \phi(t) \text { for all } t \in[\tau, T) .
$$

Thus

$$
\int_{0}^{t} \psi^{m}(s) d s \geqq(b-\varepsilon)^{m} \int_{\tau}^{t} \phi^{m}(s) d s
$$

and consequently

$$
\frac{\int_{0}^{t} \psi^{m}(s) d s}{\psi(t)} \geqq(b-\varepsilon)^{m}\left[\frac{\int_{0}^{t} \phi^{m}(s) d s}{\phi(t)}\right]\left[\frac{\psi(t)}{\phi(t)}\right]^{-1}-(b-\varepsilon)^{m} \frac{\int_{0}^{\tau} \phi^{m}(s) d s}{\psi(t)}
$$

for all $t \in[\tau, T)$. Letting $t \uparrow T$ in an appropriate fashion and using (A.1) gives

$$
\varrho \geqq(b-\varepsilon)^{m} a / b \text {. }
$$

This provides the conclusion of the lemma, since $\varepsilon \in(0, b)$ was arbitrary.

Lemma A4. Suppose that the hypotheses of Theorem 3 (iii) hold and let $\varrho$ be given by (1.9). Then $C \leqq A B^{m}$ and

$$
A B^{m-1} \chi\left(C A^{-1} B^{-m}\right) \leqq \varrho
$$

where $\chi$ is given by (1.7). 
Proof. Since

$$
\int_{0}^{t} \psi^{m}(s) d s / \psi(t)=\left[\int_{0}^{t} \psi^{m}(s) d s / \phi(t)\right] /[\psi(t) / \phi(t)]
$$

for any $t \in(0, T)$, we immediately have

$$
\varrho \geqq C / B \text {. }
$$

Moreover, given any $\varepsilon>0$ there exists a $\tau \in(0, T)$ such that (A.5) holds. Therefore

$$
\int_{0}^{t} \psi^{m}(s) d s \leqq \int_{0}^{\tau} \psi^{m}(s) d s+(B+\varepsilon)^{m} \int_{\tau}^{t} \phi^{m}(s) d s
$$

and consequently

$$
\int_{0}^{t} \psi^{m}(s) d s / \phi(t) \leqq(B+\varepsilon) \int_{0}^{t} \psi^{m}(s) d s / \psi(t)+(B+\varepsilon)^{m} \int_{0}^{t} \phi^{m}(s) d s / \phi(t)
$$

for all $t \in[\tau, T)$. Hence, letting $t \uparrow T$ and using (A.1), there holds $C \leqq(B+\varepsilon)^{m} A$ for all $\varepsilon>0$. This means $C \leqq A B^{m}$. Now, observe that the function $\chi$ is convex on $[0,1]$, $\chi(0)=0$, and $\chi(1)=1$. So, $\chi(z) \leqq z$ for all $z \in[0,1]$. In particular,

$$
\chi\left(C A^{-1} B^{-m}\right) \leqq C A^{-1} B^{-m} .
$$

Combining (A.9) and (A.10) yields (A.8).

The consistency of Theorems 3 and 4 now follows from Lemmata A2, A3, and A4, and the observation below.

Lemma A5. Let $\mu$ be given by (1.6). Then

$$
(m-1)^{-1} m^{-1}(m+1)(2 m+1) \leqq \mu \leqq 2(m-1)^{-2} m^{(2 m+1) / m}(m+1)^{1 / m} .
$$

The proof of this statement is a question of straightforward computation.

\section{Appendix B. Connection with Earlier Work}

In [3] the behaviour of solutions of problem (1.1)-(1.3) with peaking, i.e. under condition (1.4), was investigated under hypotheses (1.12) and (1.13). From this analysis it can be inferred that localization occurs if and only if (1.14) is satisfied.

The purpose of this appendix is to discuss the significance of the hypotheses $(1.12)$ and (1.13) and to reconcile the apparently different necessary and sufficient conditions for localization, viz. (1.14) deduced from [3], and, (1.5) obtained in the present paper.

Lemma B1. Suppose that (1.4), (1.12), and (1.13) hold. Then necessarily $l \leqq 0$. Furthermore:

(i) if $l<0$, given any $\varepsilon>0$ there exists a $\tau \in[0, T)$ and a $C \in(0, \infty)$ such that

$$
\psi(t) \leqq C(T-t)^{(1 / l)-\varepsilon} \text { for all } t \in[\tau, T) ;
$$

(ii) if $l>-\infty$, given any $\varepsilon>0$ there exists $a \tau \in[0, T)$ and $a C \in(0, \infty)$ such that

$$
\psi(t) \geqq C(T-t)^{1 /(l-\varepsilon)} \text { for all } t \in[\tau, T) .
$$


Proof. Hypothesis (1.13) implies that

$$
\gamma=\lim _{t \uparrow T}\left(\psi / \psi^{\prime}\right)(t)
$$

exists, and plainly $0 \leqq \gamma \leqq \infty$. We assert that

$$
\gamma=0 \text {. }
$$

Supposing that $\gamma>0$, there exists a $\delta \in(0, \infty)$ and a $\tau \in(0, T)$ such that $\left(\psi^{\prime} / \psi\right)(t)$ $<1 / \delta$ for all $t \in[\tau, T)$. Whence, integrating, $\psi(t) \leqq \psi(\tau) \exp [(t-\tau) / \delta]$ for all $t \in[\tau, T)$. Thus $\gamma$ must be equal to zero, or otherwise (1.4) is contradicted.

It follows immediately from (B.3) that $l$ is necessarily less than or equal to zero.

Now, suppose that $-\infty<l<0$. Then given any $\varepsilon>0$ there exists a $\tau \in(0, T)$ such that

$$
l-\varepsilon \leqq\left(\psi / \psi^{\prime}\right)^{\prime}(t) \leqq 1 /\{(1 / l)-\varepsilon\} \text { for all } t \in[\tau, T) .
$$

Integrating from $t \in[\tau, T)$ to $T$ and applying (B.3) subsequently yields

$$
[l-\varepsilon](T-t) \leqq-\left(\psi / \psi^{\prime}\right)(t) \leqq[1 /\{(1 / l)-\varepsilon\}](T-t) .
$$

Hence,

$$
-[1 /(l-\varepsilon)](T-t)^{-1} \leqq\left(\psi^{\prime} / \psi\right)(t) \leqq-[(1 / l)-\varepsilon](T-t)^{-1}
$$

for all $t \in[\tau, T)$. Integrating a second time verifies (B.1) and (B.2).

The assertions (B.1) and (B.2) can be deduced in the border-line cases $l=-\infty$ and $l=0$ by disregarding the irrelevant parts of the above argument.

Lemma B1 implies that if (1.12) and (1.13) hold, the boundary function $\psi(t)$ behaves almost like a power of $(T-t)$ with exponent $1 / l$, as $t \uparrow T$. Consequently, if $l \neq-(m-1)$, the results regarding localization can be straightforwardly obtained by comparison with the known similarity solutions of equation (1.1) of the form $(1.10)[5,7,8]$. It can be directly concluded that if $l<-(m-1)$, localization occurs, whereas if $l>-(m-1)$, localization is excluded.

The next lemma confirms that the conclusions drawn from the work of Galaktionov and Samarskii [3] are consistent with those of the present note, and with the preceeding observation.

Lemma B2. Suppose that (1.4), (1.12), and (1.13) hold. Let @ be given by (1.9), and

$$
\sigma=\limsup _{t \uparrow T} \psi^{m}(t) / \psi^{\prime}(t)
$$

Then $\varrho=\sigma$. Furthermore, if $l<-(m-1)$ then $\varrho=\sigma=0$, whilst if $l>-(m-1)$ then $\varrho=\sigma=\infty$.

Proof. Note that

$$
\left(\psi^{m} / \psi^{\prime}\right)^{\prime}=\psi^{m-1}\left[m-1+\left(\psi / \psi^{\prime}\right)^{\prime}\right] .
$$

Integrating, it follows from Lemma B1 that if $l<-(m-1)$ there holds $\sigma=0$, whereas if $l>-(m-1)$ there holds $\sigma=\infty$. Moreover, as a corollary of Lemma B1, one may show that $\varrho=0$ if $l<-(m-1)$. To verify the lemma, it therefore remains to show that $\varrho=\sigma$ in the event that $l \geqq-(m-1)$. 
To verify this last assertion, we start from the following variant of (B.5):

$$
\left(\psi^{m+1} / \psi^{\prime}\right)^{\prime}=\psi^{m}\left[m+\left(\psi / \psi^{\prime}\right)^{\prime}\right] .
$$

Given $\varepsilon>0$ there exists a $\tau \in(0, T)$ such that

$$
(m+l-\varepsilon) \psi^{m}(t) \leqq\left(\psi^{m+1} / \psi^{\prime}\right)^{\prime}(t) \leqq(m+l+\varepsilon) \psi^{m}(t)
$$

for all $t \in[\tau, T)$. Therefore, integrating,

$$
(m+l-\varepsilon) \int_{\tau}^{t} \psi^{m}(s) d s \leqq\left(\psi^{m+1} / \psi^{\prime}\right)(t)-\left(\psi^{m+1} / \psi^{\prime}\right)(\tau) \leqq(m+l+\varepsilon) \int_{\tau}^{t} \psi^{m}(s) d s
$$

for all $t \in[\tau, T)$. Or, rewriting,

$$
\begin{aligned}
& (m+l-\varepsilon)\left[\int_{0}^{t} \psi^{m}(s) d s / \psi(t)\right]+\left[\left(\psi^{m+1} / \psi^{\prime}\right)(\tau)-(m+l-\varepsilon) \int_{0}^{\tau} \psi^{m}(s) d s\right] / \psi(t) \\
& \leqq\left(\psi^{m} / \psi^{\prime}\right)(t) \\
& \leqq(m+l+\varepsilon)\left[\int_{0}^{l} \psi^{m}(s) d s / \psi(t)\right]+\left[\left(\psi^{m+1} / \psi^{\prime}\right)(\tau)-(m+l+\varepsilon) \int_{0}^{\tau} \psi^{m}(s) d s\right] / \psi(t)
\end{aligned}
$$

for all $t \in[\tau, T)$. In view of (1.4), under the observation $l>-m$, it is subsequently clear that $\varrho=\sigma$.

Thus, under hypotheses (1.12) and (1.13), the condition for localization deduced from the results of [3] is equivalent to those propounded in Theorem 1 of the present paper.

Note that the class of functions which satisfies (1.4), (1.12), and (1.13) with $l=-(m-1)$ covers the complete scala of values of $\varrho=\sigma$ defined by (1.9) and (B.4) respectively. Subsequently this class includes both functions for which localization does and does not occur. By way of illustration, consider

$$
\psi(t)=\psi_{0}(T-t)^{-1 /(m-1)}[-\ln (T-t)]^{p}
$$

for some $\psi_{0}>0$. This function satisfies (1.4), (1.12), and (1.13) with $l=-(m-1)$, for all real $p$. However, $\varrho=\sigma=0$ for $p<0, \varrho=\sigma=(m-1) \psi_{0}^{m-1}$ for $p=0$, whilst $\varrho=\sigma=\infty$ for $p>0$.

Acknowledgements. This work was made possible by a visit of the first author to the second author in February 1986. Gratitude is expressed to the University of Twente and to the Universidad Complutense Madrid for providing the financial support for this visit. The hospitality extended to the first author in Madrid was greatly appreciated. Furthermore, it is a pleasure to record a number of enlightening discussions with A.A. Jagers of the University of Twente concerning the relationship between the conditions in Theorems 3 and 4 which brought Appendix A to fruition.

\section{References}

1. Caffarelli, L.A., Friedman, A.: Continuity of the density of a gas flow in a porous medium. Trans. Am. Math. Soc. 252, 99-113 (1979)

2. Galaktionov, V.A., Kurdyumov, S.P., Mikhailov, A.P., Samarskii, A.A.: Asymptotic stage of regimes with peaking and effective heat localization in nonlinear heat-conduction problems. Differ. Equations 16, 743-750 (1980). Translation of: Differ. Uravn. 16, 1196-1204 (1980) 
3. Galaktionov, V.A., Samarskii, A.A.: Methods of constructing approximate self-similar solutions of nonlinear heat equations. I. Math. USSR-Sb. 46, 291-321 (1983). Translation of: Mat. Sb. (N.S.) 118, 291-322, 431 (1982)

4. Gilding, B.H.: Stabilization of flows through porous media. SIAM J. Math. Anal. 10, 237-246 (1979)

5. Gilding, B.H.: On a class of similarity solutions of the porous media equation. III. J. Math. Anal. Appl. 77, 381-402 (1980)

6. Gilding, B.H.: Improved theory for a nonlinear degenerate parabolic equation. Ann. Scuola Norm. Sup. Pisa Cl. Sci. (4) (to appear)

7. Gilding, B.H., Peletier, L.A.: On a class of similarity solutions of the porous media equation. J. Math. Anal. Appl. 55, 351-364 (1976)

8. Gilding, B.H., Peletier, L.A.: On a class of similarity solutions of the porous media equation. II. J. Math. Anal. Appl. 57, 522-538 (1977)

9. Kalashnikov, A.S.: The occurrence of singularities in solutions of the non-steady seepage equation. U.S.S.R. Comput. Math. and Math. Phys. 7, 269-275 (1967). Translation of: Zh. Vychisl. Mat. i Mat. Fiz. 7, 440-444 (1967)

10. Kurdyumov, S.P., Kurkina, E.S., Malinetskii, G.G., Samarskii, A.A.: Dissipative structures in an inhomogeneous nonlinear burning medium. Soviet Phys. Dokl. 25, 167-169 (1980). Translation of: Dokl. Akad. Nauk SSSR 251, 587-591 (1980)

11. Kurdyumov, S.P., Malinetskii, G.G., Poveshchenko, Yu.A., Popov, Yu.P., Samarskii, A.A Interaction of dissipative thermal structures in nonlinear media. Soviet Phys. Dokl. 25 252-254 (1980). Translation of: Dokl. Akad. Nauk SSSR 251, 836-839 (1980)

12. Likht, M.K.: On the propagation of perturbations in problems connected with degenerate quasilinear parabolic equations. Differential Equations 2, 496 498 (1966). Translation of: Differentsial'nye Uravneniya 2, 953-957 (1966)

13. Oleinik, O.A., Kalashnikov, A.S., Chzhou Y.-L.: The Cauchy problem and boundary problems for equations of the type of non-stationary filtration (in Russian). Izv. Akad. Nauk SSSR Ser. Mat. 22, 667-704 (1958)

14. Peletier, L.A.: The porous media equation. In: Amann, H., Bazley, N., Kirchgässner, K. (eds.) Applications of nonlinear analysis in the physical sciences, pp. 229-241. Boston: Pitman Advanced Publishing Program 1981

15. Samarskii, A.A., Elenin, G.G., Zmitrenko, N.V., Kurdyumov, S.P., Mikhailov, A.P.: The burning of a nonlinear medium in the form of complex structures. Soviet Phys. Dokl. 22 , 737-739 (1977). Translation of: Dokl. Akad. Nauk SSSR 237, 1330-1333 (1977)

16. Samarskii, A.A., Galaktionov, V.A., Kurdyumov, S.P., Mikhailov, A.P.: Localization of diffusion processes in media with constant properties. Soviet Phys. Dokl. 24, 543-545 (1979). Translation of: Dokl. Akad. Nauk SSSR 247, 349-353 (1979)

17. Samarskii, A.A., Zmitrenko, N.V., Kurdyumov, S.P., Mikhailov, A.P.: Effect of metastable localization of heat in a medium with nonlinear thermal conductivity. Soviet Phys. Dokl. 20, 554-556 (1976). Translation of: Dokl. Akad. Nauk SSSR 223, 1344-1347 (1975)

18. Samarskii, A.A., Zmitrenko, N.V., Kurdyumov, S.P., Mikhailov, A.P.: Thermal structures and fundamental length in a medium with nonlinear heat conduction and volumetric heat sources. Soviet Phys. Dokl. 21, 141-143 (1976). Translation of: Dokl. Akad. Nauk SSSR 227, 321-324 (1976)

19. Sattinger, D.H.: On the total variation of solutions of parabolic equations. Math. Ann. 183, $78-92$ (1969)

20. Stokes, A.N.: Intersections of solutions of nonlinear parabolic equations. J. Math. Anal. Appl. 60, 721-727 (1977)

21. Zel'dovich, Ya.B., Raizer, Yu.P.: Physics of shock waves and high-temperature hydrodynamic phenomena. Vol. I: New York: Academic Press 1966. Vol. II: New York: Acadenic Press 1967 